\title{
INVESTIGACIONES
}

\section{MODELO CONCEPTUAL PARA LA DESERCION ESTUDIANTIL UNIVERSITARIA CHILENA}

\author{
Conceptual model for dropout chilean university student
}

\author{
Christian Díaz Peralta \\ Universidad Católica de la Santísima Concepción, \\ Código Postal: 4090541. \\ chdiaz@ucsc.cl
}

\section{Resumen}

Este estudio propone un modelo conceptual que explica la deserción/permanencia como resultado de la motivación (positiva o negativa), la que es afectada por la integración académica y social. A su vez, éstas están compuestas por las principales características preuniversitarias, institucionales, familiares, individuales y las expectativas laborales. Además, se propone un modelo conceptual del equilibrio dinámico del estudiante de su permanencia en la universidad al combinar los factores académicos, sociales e institucionales, el que permite ilustrar cómo el estudiante debe adaptarse a los cambios en las tensiones que se producen entre los distintos factores que lo afectan.

Palabras clave: Deserción estudiantil, permanencia académica, educación superior.

\begin{abstract}
This study proposes a conceptual model that explains the drop/retention as a result of the motivation (positive or negative), which is affected by the academic and social integration. In turn, they are composed of the main features of high school education institutional, family and individual job prospects. In addition, we propose a conceptual model of the dynamic balance of student retention in college by combining academic, social, and institutional factors which illustrates how the student must adapt to changes in the tensions that arise between different factors affecting it.
\end{abstract}

Key words: student dropout, academic retention, higher education. 


\section{INTRODUCCION}

La deserción estudiantil es uno de los problemas que aborda la mayoría de las instituciones de educación superior de toda Latinoamérica. A través de distintas investigaciones, se da cuenta de un número importante de estudiantes que no logran culminar sus estudios universitarios, con el consecuente costo social asociado a este fenómeno. Por lo anterior, algunos países han comenzado a diseñar profundos procesos de mejoramiento para aumentar la retención en los primeros años de estudios universitarios (UNESCO 2004).

En Chile, de acuerdo a estimaciones basadas en estadísticas nacionales, la tasa de deserción global de pregrado, evaluada para el año 2004, es cercana al 53,7\%, siendo mayor en las universidades privadas nuevas que en las públicas. Por su parte, las áreas del conocimiento más críticas son Humanidades y Derecho con cifras del orden de $80 \%$, y las más eficientes son las áreas de Educación y Salud con un 37\% y un 27\%, respectivamente. Por género, las mujeres poseen una tasa de deserción promedio más baja que los varones de $43 \%$ y 50\%, respectivamente (González 2005).

Para el sistema nacional el efecto de la deserción en términos de costos es de 96,2 millones de dólares. De acuerdo a esta cifra, el gasto por abandono es equivalente al $26 \%$ del gasto público en educación universitaria, mientras que representa el 10,2\% del gasto total (público y privado) en el sector universitario (UNESCO 2004).

Por estos impactos sociales y económicos mencionados anteriormente, la deserción estudiantil universitaria se ha trasformado en uno de los aspectos a evaluar por el Ministerio de Educación de Chile, en las universidades que reciben aportes del Estado. Si bien el modelo de asignación que rige el Aporte Fiscal Directo incorpora ciertos indicadores, en el aporte variable del $5 \%$ éstos no reflejaban la eficiencia de los procesos internos de las instituciones, como lo es la retención. Sin embargo, desde el año 2006 el sistema de financiamiento estudiantil a través del Arancel de Referencia y de la Ley No 20.027, incorporó la deserción estudiantil como indicador de evaluación institucional.

Considerando la necesidad de describir el fenómeno de la deserción para diseñar acciones que propendan a la disminución de la deserción, el presente estudio tiene como propósito determinar un modelo conceptual que explique la deserción estudiantil universitaria de pregrado en el sistema universitario chileno. Este trabajo se estructura en tres partes, la primera da cuenta del marco teórico acerca de la deserción, desde la conceptualización del problema hasta la síntesis de los principales modelos teóricos, la segunda proporciona la descripción del método para el análisis de la información y la caracterización de las variables y, en la última, las conclusiones y extensiones.

\section{MARCO TEORICO}

2.1. LA EDUCACION SUPERIOR EN CHILE. El sistema de educación superior nacional en el año 2005 estaba compuesto por 181 instituciones, de las cuales 60 (33\%) eran universidades, de éstas 25 (14\%) correspondían al Consejo de Rectores de Universidades Chilenas (CRUCH) y $35(19 \%)$ eran universidades privadas, $37(20 \%)$ institutos profesionales, 69 (38\%) centros de formación técnica y 15 (9\%) instituciones de estudios superiores de las Fuerzas Armadas y de Orden (I.N.D.I.C.E.S. 2006). 
De acuerdo al modelo de financiamiento de la educación superior publicado por Dohmen (2002), la principal modalidad que Chile posee es la guiada por la demanda y se dirige a financiar insumos; por ejemplo, mediante vouchers, donde el financiamiento de las instituciones se realiza a través de la elección realizada por los clientes o consumidores. El voucher puede ser sustituido o complementado, siguiendo la lógica de soberanía del consumidor, por un sistema de becas y créditos estudiantiles, alcanzándose el mismo objetivo, éste es, que los clientes o consumidores se hagan cargo, a través de sus opciones, de controlar la calidad del servicio educacional provisto por las instituciones.

Según Brunner (2006), estamos frente a una estructura de la educación superior chilena altamente privatizada en el contexto internacional comparado. La mayor parte de los recursos que ingresan al sistema proviene de las familias y de los propios alumnos, y se canaliza a través de una modalidad descentralizada y dirigida a insumos. Sí se incluye allí, además de las diferentes modalidades del crédito estudiantil, el pago directo de aranceles por parte de los usuarios del servicio. Esta última característica del sistema chileno refuerza su tipificación como un sistema en avanzada fase de privatización y mercadización (Brunner 2005). Así es como desde la organización de la economía política del sistema y el uso intensivo de instrumentos de tipo mercado, para asignar recursos públicos a las universidades, prevalece un esquema descentralizado de oferta, con un gobierno que actúa a la distancia. Según Bernasconi y Rojas (2004) estiman que todo el costo anual en educación e investigación para el erario público es de aproximadamente US\$ 488 millones, lo que equivale a US\$ 897 por estudiante.

En consecuencia, el sistema se configura desde un modelo de competencia, sin una coordinación planificada centralizada. En los últimos años, la autoridad pública ha profundizado componentes de competencia, al mismo tiempo propende regular sus efectos en función de objetivos de equidad (créditos estudiantiles), calidad (exigencias de información y acreditación) y desarrollo de capacidades (a través de la asignación competitiva de recursos a las instituciones y para la investigación) (Brunner 2005).

2.2. LA DESERCION UNIVERSITARIA EN CHILE. En Chile la deserción universitaria no ha sido suficientemente investigada ni se cuenta con una base estadística por instituciones a nivel nacional; sin embargo, el Ministerio de Educación ha mostrado preocupación por los efectos del fenómeno de la deserción. En una reciente investigación, dirigida por el Consejo Superior de Educación (CSE) sobre la base de la información recopilada en I.N.D.I.C.E.S. (2006), se afirma que las universidades del CRUCH tienen mayor retención de su alumnado, manteniendo al $73 \%$ de ellos al finalizar el segundo año, a diferencia de las instituciones privadas que mantienen sólo al 65\%. Otra conclusión del estudio revela que los alumnos provenientes de colegios municipalizados conforman la mayoría del total que ingresa a la educación superior; son éstos los que presentan los mayores indicadores de deserción en todo el sistema de educación terciaria.

Según González (2005), la deserción tiene consecuencias sociales en términos de las expectativas de los estudiantes y sus familias; emocionales por la disonancia entre las aspiraciones de los jóvenes y sus logros y, también, importantes consecuencias económicas tanto para las personas como para el sistema en su conjunto. Adicionalmente, quienes no concluyen sus estudios se encuentran en una situación de empleo desfavorable respecto a quienes terminan. Algunos estudios estiman una diferencia en más de 
un $45 \%$ en los salarios a favor de quienes terminan sus estudios universitarios, respecto de quienes no lo hacen.

En el país, son escasos los estudios que han intentado modelar y estimar las causas por las cuales un estudiante decide abandonar sus estudios superiores, y con esto formular políticas y mecanismos de reingeniería educativa que permitan la permanencia de los mismos dentro del sistema de educación superior o de la institución universitaria (González 2005; Donoso y Schiefelbein 2007). Lo que prevalece en estos estudios es la caracterización de la población desertora, la construcción de indicadores de eficiencia y efectividad del proceso formativo y la propia estadística descriptiva del fenómeno a través de las distintas cohortes.

Los estudios que existen, excepto González y Uribe (2002) y González (2005), son análisis de casos de instituciones y el abordaje metodológico ha sido de tipo estático (Casanova 2005; Gómez et al. 2002; Mella et al. 2002; Prieto 2002), sin considerar la evolución del fenómeno en el tiempo. Otro problema que enfrentan los estudios en países como Chile es la calidad de los datos, en cuanto a confiabilidad y oportunidad. Cuando se tienen datos de baja confiabilidad no es posible la comparabilidad (Salinas y Llanes 2003; Herzog 2004; Girón y González 2005, e Ishitani y Sinder 2006).

2.3. LA DESERCION DESDE LA PERSPECTIVA INSTITUCIONAL. Actualmente, a pesar de que la definición de deserción estudiantil está en discusión, existe consenso en definirla como un abandono voluntario que puede ser explicado por diferentes categorías de variables: socioeconómicas, individuales, institucionales y académicas. Sin embargo, la forma de operacionalizar las mismas depende del punto de vista desde el cual se haga el análisis; esto es, individual, institucional y estatal o nacional. Tinto (1989) afirma que el estudio de la deserción en la educación superior es extremadamente complejo, ya que implica no sólo una variedad de perspectivas, sino que, además, una gama de diferentes tipos de abandono. Adicionalmente, afirma que ninguna definición puede captar en su totalidad la complejidad de este fenómeno, quedando en manos de los investigadores la elección de la definición que mejor se ajuste a sus objetivos y al problema a investigar.

Desde el punto de vista institucional, todos los estudiantes que abandonan su educación superior pueden ser clasificados como desertores. Es así como varios autores asocian la deserción con los fenómenos de 'mortalidad' académica y retiro forzoso. En este sentido, cada estudiante que abandona la institución crea un lugar vacante que pudo ser ocupado por otro alumno que permaneciera en sus estudios, por lo cual la pérdida de estudiantes causa serios problemas financieros a las instituciones al producir inestabilidad en la fuente de sus ingresos (Tinto 1989). Sin embargo, no es claro que todos los tipos de abandono requieran la misma atención o exijan similares formas de intervención por parte de la institución, siendo ésta la gran dificultad que enfrentan las instituciones educativas. El conocimiento de estas diferencias constituye la base para elaborar políticas universitarias eficaces con el fin de aumentar la retención estudiantil.

2.3.1. Definición teórica y práctica de la deserción. Tinto (1982) define deserción como una situación a la que se enfrenta un estudiante cuando aspira y no logra concluir su proyecto educativo. Luego, es posible considerar como desertor a aquel individuo que siendo estudiante de una institución de educación superior no presenta actividad académica durante tres semestres académicos consecutivos. En algunas investigaciones, 
a este comportamiento se le denomina 'primera deserción' (first drop-out), ya que no se puede determinar si pasado este periodo de tiempo el individuo retomará o no sus estudios o si decidirá iniciar otro programa académico (Tinto 1989; Cabrera et al. 1992 y 1993; Adelman 1999).

Según González (2005), se pueden diferenciar dos tipos de abandonos en los estudiantes universitarios, con respecto al tiempo (inicial, temprana y tardía) y con respecto al espacio (institucional, interna y del sistema educativo).

\subsubsection{La deserción a través de la trayectoria académica. Según Tinto (1989), existen} varios periodos críticos en la trayectoria estudiantil en que las interacciones entre la institución y los estudiantes pueden influir directamente en la deserción. El primero, se desarrolla durante el proceso de admisión, cuando el estudiante realiza el primer contacto con la universidad. Durante la etapa de indagación de los requisitos para ingresar a una determinada institución, los estudiantes forman las primeras impresiones sobre las características sociales e intelectuales de la misma. Estas impresiones se originan en gran medida en los mensajes que se distribuyen a los potenciales postulantes en el proceso de admisión, a través de los distintos medios de comunicación, tales como: folletería, televisión, radioemisoras, internet, entre otros. Esto contribuye a crear expectativas sobre la naturaleza de la vida institucional previo a su ingreso, y esas expectativas influyen en la calidad de las primeras interacciones que se establecen con la institución. La formación de expectativas sobre las condiciones de la vida estudiantil o académica puede conducir a decepciones tempranas en el caso que lo esperado sea mayor que lo recibido. Ello pone en movimiento una serie de interacciones que lleven a la deserción. Por lo tanto, es fundamental que la institución, a través de los distintos canales de comunicación o medios publicitarios, genere expectativas realistas y precisas acerca de las características de la vida institucional en los estudiantes que ingresan.

Una segunda etapa crítica en la trayectoria académica del estudiante es la de transición entre la enseñanza media y la institución, inmediatamente después del ingreso a la institución (Montes 2002). En el primer semestre, en particular en las primeras seis semanas, se pueden presentar grandes dificultades. Esto se presenta en las grandes universidades, porque los estudiantes son obligados a transitar desde el ambiente conocido y relativamente seguro del colegio, al mundo en apariencia impersonal de la universidad, en el cual deben valerse por sí mismos, tanto en el aula como en los distintos espacios institucionales. La rapidez y el grado de la transición plantean serios problemas en el proceso de ajuste a muchos estudiantes que no son capaces de cumplir en forma independiente. La sensación de estar 'perdido' o de no ser capaz de establecer contacto con otros miembros de la institución expresa, en parte, la situación anímica en que se encuentran muchos estudiantes.

El problema de la transición en la universidad es común para una diversidad de estudiantes, no sólo para aquellos que pasan desde la enseñanza media a una institución de educación terciaria, con grandes espacios para la vida académica, sino que, también, es una cuestión no menos importante, en aquellas instituciones con gran cantidad de estudiantes de generaciones anteriores. Para el estudiante que ingresa a la universidad y lleva más de un año fuera del sistema terciario puede resultar traumática la transición entre el entorno del hogar o del trabajo y el ambiente juvenil de la institución (Tinto 1989). Los problemas originados en la transición pueden ser igualmente severos para 
jóvenes provenientes de comunas rurales y pertenecientes a estratos socioeconómicos bajos, ya que poseen condiciones económica y sociales, en términos relativos, desventajosas al ingresar a una universidad o carrera que posee mayoritariamente estudiantes pertenecientes a los estratos superiores.

Durante el periodo de transición el abandono es más frecuente en la última fase del primer año de estudios y antes del comienzo del segundo (Tinto 1989). Mientras algunos de estos abandonos se originan debido a la incapacidad de satisfacer exigencias académicas, la mayor parte son deserciones voluntarias. En tal situación, es más frecuente que los estudiantes se inscriban en otras instituciones a que abandonen en forma permanente el sistema de educación terciaria. Son numerosos los motivos para ese comportamiento. Algunos estudiantes deciden que las exigencias de la vida académica no son congruentes con sus intereses y preferencias; otros tienen dificultades para lograr relaciones en los ambientes académico y social de la universidad, y aun hay estudiantes que prefieren no establecer esas relaciones, porque encuentran que las características de la comunidad institucional son inapropiadas para sus escalas de valores y afinidades sociales; en tanto que cierto número de sujetos son incapaces de tomar decisiones sobre la forma en que deben dirigir sus energías y recursos, otros llegan a la conclusión de que completar estudios universitarios no constituye una meta deseable (Tinto 1989).

2.4. MODELOS DE ANALISIS DE LA DESERCION ESTUDIANTIL. La deserción se puede explicar como el resultado de distintos vectores que afectan al estudiante. Por ello, es necesario estudiar y analizar directamente al desertor, ya que la deserción es un fenómeno inherente a la vida estudiantil que seguramente se mantendrá, por estar relacionado a procesos dinámicos de selección, rendimiento académico y de la eficiencia del sistema educativo en general. A continuación, se describen algunas teorías sobre la deserción que permiten enfocar el problema desde distintas perspectivas.

2.4.1. Modelos psicológicos. Estos señalan que los rasgos de la personalidad son los que diferencian a los estudiantes que terminan sus estudios regulares de aquellos que no lo logran. Fishbein y Ajzen (1975) proponen la Teoría de la Acción Razonada que analiza el comportamiento como actitudes en respuesta a objetos específicos, considerando normas subjetivas que guían el comportamiento hacia esos objetos y el control percibido sobre ese comportamiento. Estos autores señalan que la 'intención de tomar la acción' es determinada por dos factores: primero; 'actitud hacia tomar la acción', y segundo la 'norma subjetiva'. La norma subjetiva se refiere a cómo se espera que el individuo se comporte en la sociedad, la cual es determinada por una evaluación de la expectativa. En el caso de la decisión de desertar o permanecer se ve influida por: conductas previas, actitud acerca de la deserción o permanencia y normas subjetivas acerca de estas acciones. En consecuencia, según estos autores la deserción es el resultado del debilitamiento de las intenciones iniciales.

Asimismo, Ethington (1990) examinó la validez del Modelo de Elección Académica (MEA) de Eccles et al. (1984), el cual se basa en diversos marcos teórico-empíricos (toma de decisiones, motivación al logro y teorías de la atribución). Ethington (1990) introduce una teoría más general sobre las conductas de logro, y con ello concluye que el rendimiento académico previo afecta el desempeño futuro al actuar sobre el 
autoconcepto del estudiante, su percepción de las dificultades de estudio, sus metas, valores y expectativas de éxito.

Otro modelo propuesto es el de Bean y Eaton (2001), cuyas bases fundamentales fueron los procesos psicológicos relacionados con la integración académica y social. Estos autores presentaron cuatro teorías psicológicas como línea de base del modelo: (a) Teoría de actitud y comportamiento, la que proviene de la estructura de su modelo; (b) Teoría del comportamiento de copia, la habilidad para entrar y adaptarse a un nuevo ambiente; (c) Teoría de autoeficacia, una percepción individual capaz de tratar con tareas y situaciones específicas; (d) Teoría de atribución, donde un individuo tiene un fuerte sentido de control interno. Estos autores enfatizaron la importancia que posee la institución al disponer de servicio de enseñanza, comunidades de aprendizaje al primer año, seminarios de orientación profesional y programas de mentoring para apoyar el éxito de los estudiantes.

2.4..2. Modelos sociológicos. Los modelos sociológicos enfatizan respecto a la influencia en la deserción de factores externos al individuo, adicionales a los psicológicos. Spady (1970) se basó en el modelo suicida de Durkheim (1951) indicando que la deserción es el resultado de la falta de integración de los estudiantes en el entorno de la educación superior; aduce que el medio familiar es una de las muchas fuentes que expone a los estudiantes a influencias, expectativas y demandas, las que a su vez afectan su nivel de integración social en la universidad; la congruencia normativa actúa directamente sobre el rendimiento académico, el desarrollo intelectual, el apoyo de pares y la integración social. Este autor señala que si las influencias señaladas no se producen en la dirección positiva, implica rendimiento académico insatisfactorio, bajo nivel de integración social y de satisfacción y compromiso institucional y una alta probabilidad de que el estudiante decida abandonar sus estudios.

Este autor encontró seis predictores de la deserción estudiantil en college's norteamericanos: integración académica, integración social, estado socioeconómico, género, calidad de la carrera y el promedio de notas en cada semestre ${ }^{1}$.

2.4.3. Modelos económicos. Según las investigaciones desarrolladas por Cabrera et al. (1992 y 1993), Bernal et al. (2000) y St. John et al. (2000) se pueden distinguir dos modelos: (1) Costo/Beneficio: consiste en que cuando los beneficios sociales y económicos asociados a los estudiantes son percibidos como mayores que los derivados por actividades alternas, como por ejemplo un trabajo, por lo cual el estudiante opta por permanecer en la universidad ${ }^{2}$ y (2) Focalización de Subsidio: consiste en la entrega de subsidios que constituyen una forma de influir sobre la deserción. Estos subsidios están

1 Otro autor que planteó un modelo basado en la teoría de la participación fue Astin (1999), quien en su investigación destaca la relación entre variables de las teorías pedagógicas tradicionales (recursos didácticos, contenidos de materias y en enfoque de la enseñanza) y los resultados del aprendizaje deseado por el estudiante y el profesor.

2 Un componente crítico de este modelo es la percepción que tiene el estudiante de su capacidad o incapacidad de solventar los costos asociados a los estudios universitarios. 
dirigidos a los grupos que presentan limitaciones reales para costear sus estudios ${ }^{3}$. Este modelo busca privilegiar el impacto efectivo de los beneficios estudiantiles por sobre la deserción, dejando de lado las percepciones acerca de la adecuación de dichos beneficios o el grado de ajuste de éstos a los costos de los estudios (Himmel 2002) . $^{4}$

Las ayudas proporcionadas a los estudiantes en forma de becas constituyen un factor de peso en las posibilidades de permanencia, observándose que las tasas de deserción varían dependiendo de la cantidad y duración de la ayuda financiera con la que cuenta el estudiantado (Ishitani y DesJardins 2002) e inclusive ante apuros económicos se provoca un impacto importante en el abandono temprano (Ozga y Sukhmandan 1998).

2.4.4. Modelo organizacional. Este modelo de análisis sostiene que la deserción depende de las cualidades de la organización en la integración social, y más particularmente en el abandono de los estudiantes que ingresan a ella (Berger y Milem 2000; Berger 2002; Kuh 2002). En este enfoque es altamente relevante la calidad de la docencia y de la experiencia de aprender en forma activa por parte de los estudiantes en las aulas, las cuales afectan positivamente la integración social del estudiante (Braxton et al. 1997).

2.4.5. Modelo de interacción. Tinto (1975) explica el proceso de permanencia en la educación superior como una función del grado de ajuste entre el estudiante y la institución, adquirido a partir de las experiencias académicas y sociales (integración). Este autor expande el modelo de Spady (1970), incorporando la teoría de intercambio de Nye (1976). La base de la teoría de intercambio se encuentra en el principio de que los seres humanos evitan las conductas que implican un costo de algún tipo para ellos y buscan recompensas en las relaciones, interacciones y estados emocionales. Tinto (1975) afirma que los estudiantes actúan de acuerdo con la teoría de intercambio en la construcción de su integración social y académica, es decir, si el estudiante percibe que los beneficios de permanecer en la universidad son mayores que los costos personales, entonces el estudiante permanecerá en la institución. En el sentido contrario, si percibe que otras actividades son percibidas como fuentes de recompensas más altas, el estudiante tenderá a desertar. Tinto (1987) sugiere que una buena integración es uno de los aspectos más importantes para la permanencia, y que esta integración depende de: las experiencias durante la permanencia en la universidad, las experiencias previas al acceso universitario y las características individuales que, por otro lado, son susceptibles a las políticas y prácticas universitarias.

Posteriormente, Tinto $(1989,1993)$ profundiza el análisis del modelo inicial del año 1975 y en esta oportunidad se observa que a medida que el estudiante avanza en

3 En este modelo, aunque se incluyen las variables demográficas y académicas, éstas sólo se incorporan en el análisis como forma de controlar las fuertes variaciones que pueden enmascarar los efectos directos de los subsidios y los costos de los estudios sobre la retención de estudiantes.

$4 \quad$ Una derivada de estos modelos es el economicista, el cual explica la deserción como la elección por parte del estudiante de una forma alternativa de invertir tiempo, energía y recursos que puedan producirle en el futuro beneficios mayores respecto a los costos de permanencia en la universidad (Cabrera et al. 2006). Este planteamiento se basa en la teoría del capital humano, según la cual un estudiante invertirá tiempo y recursos monetarios en educación, solamente si los beneficios que obtiene son suficientes para cubrir los costos de la educación y si la educación superior es al menos tan rentable como los usos alternativos de esos mismos recursos. 
su trayectoria académica, diversas variables contribuyen a reforzar su adaptación a la institución que seleccionó, ya que ingresa a ella con un conjunto de características que influyen sobre su experiencia, en la educación terciaria. Estas características comprenden antecedentes familiares, tales como los valores que ésta sustenta, además de sus atributos personales y de la experiencia académica preuniversitaria. Todos estos aspectos relevantes se combinan para influir sobre el compromiso inicial con la institución, así como para la consecución de su meta, que es la graduación o titulación.

Los estudios que han empleado el modelo de Tinto confirman la capacidad predictiva directa de la integración social sobre la deserción, sobre todo en las universidades tradicionales. Sin embargo, algunos estudios han puesto en duda si el modelo del Tinto es aplicable a todos los estudiantes e instituciones de educación superior. Estos estudios indican que si bien la integración social y académica son factores importantes para predecir la persistencia, no tienen la misma importancia en todos los estudiantes. Anderson (1981), Braxton y Brier (1989), Pascarella y Chapman (1983a, 1983b) y Pascarella et al. (1986), Pascarella y Terenzini (1985) reportaron que la integración académica es más fuerte que los compromisos o metas institucionales; por tanto, los compromisos institucionales tienen un efecto indirecto sobre la permanencia a través de la integración social ${ }^{5}$.

Bean (1980) incorpora al modelo de Tinto (1975) las características del modelo de productividad desarrollado en el contexto de las organizaciones laborales. Reemplaza las variables del ambiente laboral por las más adecuadas al entorno de la educación superior, de tal forma que la satisfacción con los estudios es similar a la satisfacción con el trabajo. Sostiene que inciden en la deserción los siguientes factores: (1) Factores Académicos: preuniversitarios, integración académica y desempeño académico; (2) Factores Psicosociales: metas, utilidad percibida, interacción con pares y docentes; (3) Factores ambientales: financiamiento, oportunidad de transferirse, relaciones sociales externas; (4) Factores de socialización: rendimiento académico, adaptación y compromiso institucional.

En estudios más recientes Bean y Vesper (1990) diseñaron un modelo para explicar el proceso de la persistencia en los college's norteamericanos que se basa, predominantemente, en factores externos a la institución. Las variables consideradas se relacionan con la institución, la persona o el ambiente; es decir, el ámbito exterior a la institución. Consideraron que estos factores también tienen un peso significativo en la deserción, en particular en la deserción voluntaria.

2.5. E-LEARNING Y LA DESERCION ESTUDIANTIL. Si bien las investigaciones acerca de la deserción estudiantil en la educación a distancia no son algo nuevo, el estudio de la retención de aprendizaje electrónico es un ámbito relativamente nuevo para la investigación. Como se analizó anteriormente, los modelos existentes de permanencia o deserción se basan en investigaciones basadas en campos tradicionales de los procesos de enseñanza y aprendizaje que desarrollan los profesores y estudiantes, respectivamente. Autores como Roberts (1984), Pascarella (1985), Sweet (1986), Powell et al. (1990) y Carr (2000)

5 Investigaciones similares señalan que la mayoría de los estudiantes que desertan de la universidad no valoran la integración social como un factor importante al momento de decidir la permanencia o abandono (Andrés et al. 1996; Bean y Metzner 1985; Benjamin y Hollings 1995; Ethington 1990; Johnson 1991; Voorhees 1987). 
señalan que el fenómeno de la deserción estudiantil en la educación a distancia es de naturaleza multidimensional, altamente complejo y en constante evolución.

Al respecto, Bean y Metzner (1985) desarrollaron un modelo de deserción estudiantil para estudiantes cuyo régimen de formación es a través de la enseñanza vía electrónica (e-learning $)^{6}$. Dado que estos estudiantes no presenciales no tienen la posibilidad de ser socialmente integrados en la institución, se buscó explicar el fenómeno a través de otros factores. Este modelo reconoce el bajo aporte que presenta la integración social de la eliminación natural de los estudiantes explicando que la decisión de abandono del estudiante se basa principalmente en cuatro conjuntos de variables: (a) del rendimiento académico medido por el valor promedio de notas (Grade Point Average, GPA); (b) la intención de abandonar influenciada principalmente por los resultados psicológicos y variables académicas; (c) experiencia académica previa, sobre todo de rendimiento escolar y metas educativas, y (d) las variables ambientales, que se espera que tengan importantes efectos directos en las decisiones de abandono.

Los factores externos, la motivación y las percepciones de la enseñanza a distancia muestran relaciones en los estudiantes al tomar las decisiones de abandono o de permanencia (Wang y Wong 2004), presentando los estudiantes adultos mayor compromiso con las metas Kember (1989). Sin embargo, Frankola (2001) encontró que la falta de supervisión, motivación, problemas con la tecnología, falta de apoyo a los estudiantes, las preferencias de aprendizaje, diseño inadecuado de los cursos e instructores inexpertos, son las principales causas que explica la deserción.

Wylie (2005) propone que la deserción es un proceso cíclico de decisiones de corto plazo, donde el estudiante permanentemente está realizando ajustes académicos y sociales, a partir de los resultados de las evaluaciones académicas. De manera continua los conceptos asociados a la integración académica y social están afectados negativamente, el estudiante reevalúa su permanencia en función del incremento de separación de las pautas de comportamiento, por ejemplo, la asistencia esporádica. Este proceso es teorizado como una espiral natural y continua hasta la separación del compromiso con sus estudios.

\section{METODOLOGIA}

3.1. Diseño de un modelo conceptual de deserción estudiantil. Con el objeto de comprender el fenómeno de deserción estudiantil incorporando los diversos factores que participan en él, se procede, mediante un enfoque holístico, a diseñar un modelo que explique la deserción. Para ello se desarrollan dos etapas:

Etapa 1: Sistematización de la información. Para lograr la sistematización de la literatura disponible sobre deserción estudiantil, se utilizó una matriz topológica (Collen y Gasparski 1995). Esta matriz permite presentar en un eje vertical los principales autores y a continuación un ordenamiento de las variables utilizadas según categorías (socioeconómicas, individual, académica e institucional). A partir de este eje, por cada

6 Se basaron en los modelos teóricos de Spady (1970), Tinto (1975) y Pascarella (1985) enfatizando en la integración social para explicar la eliminación natural de los estudiantes. 
punto (autor o categoría) se trazan diagonales, lo que permite intersectar las variables con los autores y entre variables presenten su relación.

De la matriz obtenida, se observaron los tipos de relaciones entre autores y categorías de variables analizadas.

Etapa 2: Propuesta de Modelo Conceptual. Se propone presentar gráficamente un modelo generalizado de deserción estudiantil. Con este modelo se busca representar los factores relevantes que participan en la deserción del estudiante. Luego, se representa en forma gráfica al estudiante, su dinámica con los factores y su condición de equilibrio y cambio del mismo.

En este estudio el criterio de selección de las variables se basó principalmente en los antecedentes reportados por la literatura (Astin 1975; Bean 1982; Bean y Metzner 1985; Cabrera et al. 1992 y 1993; Canales y De los Ríos 2007; Ethington 1990; Pascarrella y Terenzini 1980; Pascarella y Terenzini 1991; Spady 1970; St. John et al. 2000; Tinto 1975, 1993) y en la información que poseen las universidades pertenecientes al $\mathrm{CRUCH}^{7}$.

\section{RESULTADOS}

4.1. SISTEMATIZACION DE LA LITERATURA REFERIDA A LA DESERCION ESTUDIANTIL. La revisión de la literatura permitió identificar distintos enfoques de cómo abordar este fenómeno de la deserción que viven las universidades del país. Al efecto, en la figura 1 se presenta la revisión bibliográfica a través de la matriz topológica. En ella se grafica la relación de cada autor con las categorías y variables utilizadas en su investigación. Además, se muestra la relación entre autores en el desarrollo del conocimiento asociado a la deserción o permanencia estudiantil.

Los autores que se presentan en la figura 1 son los que tienen un mayor impacto en el desarrollo de modelos teóricos sobre la deserción. Los autores se ordenaron en forma cronológica. Las variables que presentan una mayor frecuencia en los estudios se agrupan en cuatro categorías: individuales (edad, género, grupo familiar e integración social), académicas (orientación profesional, desarrollo intelectual, rendimiento académico, métodos de estudios, procesos de admisión, grados de satisfacción de la carrera y carga académica), institucionales (normativas académicas, financiamiento estudiantil, recursos universitarios, calidad del programa o carrera y relación con los profesores y pares) y socioeconómicas (estrato socioeconómico, situación laboral del estudiante, situación laboral de los padres y nivel educacional de los padres).

La matriz topológica permite hacer una lectura integrada relacionando: autor-autor, autor-variables y variables-variables. A modo de ejemplo, uno de los autores más referenciados en los distintos estudios -y cuyo modelo de análisis ha sido reconocido a nivel

Las variables relacionadas con las categorías individual y socioeconómica se obtienen desde la base de datos del Departamento de Evaluación, Medición y Registro Educacional de la Universidad de Chile (D.E.M.R.E.). Las variables de la categoría académica se obtienen desde los sistemas de administración del avance académico de los estudiantes que posee cada universidad y del D.E.M.R.E.; y las institucionales desde los sistemas de gestión financiera central que poseen las universidades del CRUCH. 
mundial- es Tinto (1975). Esto se aprecia si el lector se ubica sobre el autor y baja por la diagonal; intersectará a los autores que han utilizado como base el modelo generado por Tinto, caracterizado por el ícono 'Relación entre autores' señalado en el recuadro de la figura, por ejemplo, Bean (1980 y 1982), Pascarella (1985) y el último Braxton et al. (1997); si se continúa bajando por la diagonal, se intersectará con las diagonales que suben desde las variables agrupadas según categorías, y así se muestra qué variables utilizó este autor en su estudio, como fue en este caso la edad, género, grupo familiar e integración social (identificadas con el ícono del recuadro 'Categoría individual'), y así sucesivamente con las categorías académica, institucional y socioeconómica.

\section{Figura 1}

Matriz topológica de la sistematización de la literatura relacionada con la deserción estudiantil

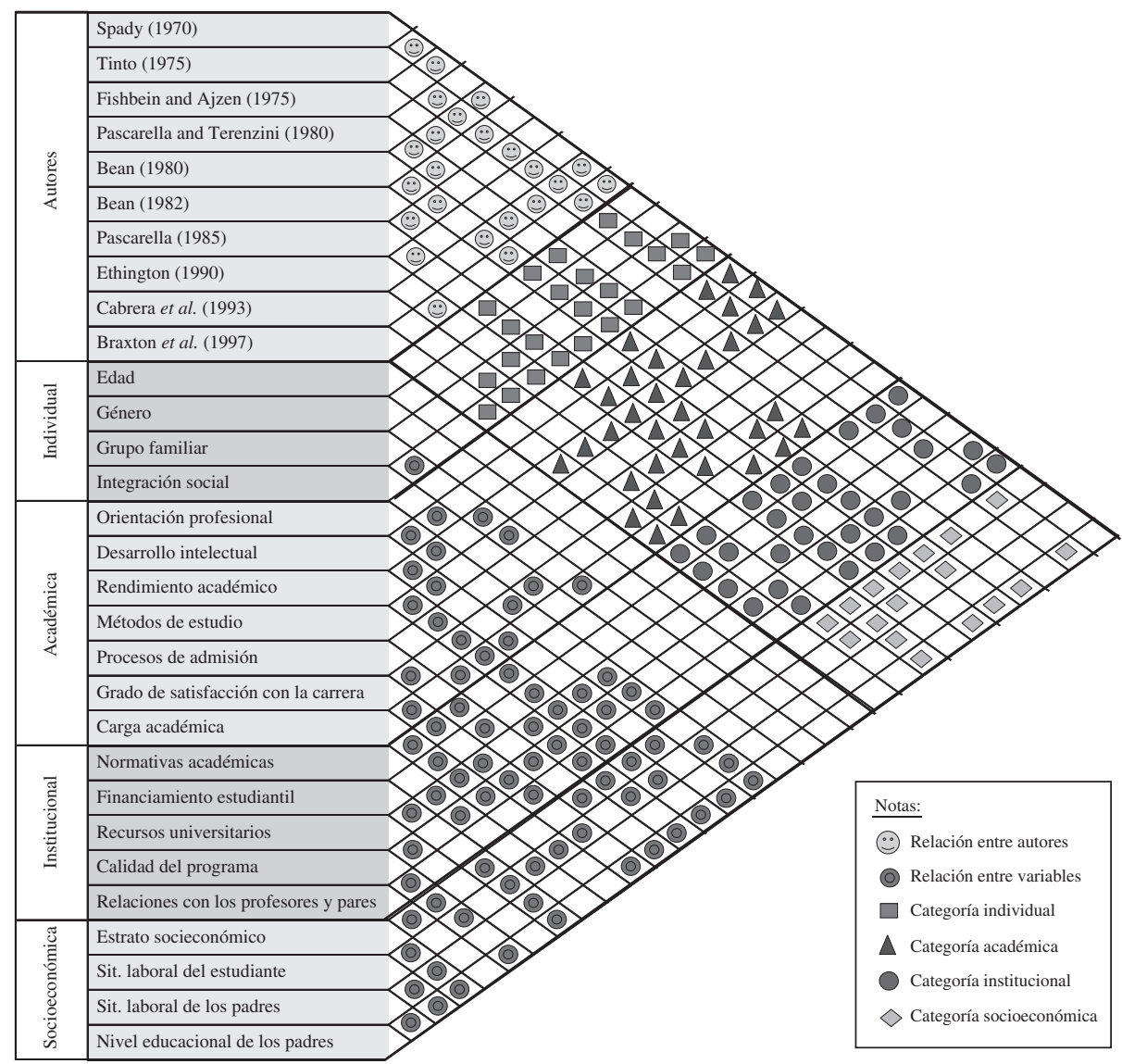

Fuente: Elaboración propia. 
Otra lectura que permite la matriz topológica es observar relaciones entre las variables para las distintas categorías. Por ejemplo, si se considera dentro de la categoría académica la variable Orientación profesional, y se baja por su diagonal, ésta se interceptará en la misma categoría bajando hacia la izquierda con la variable Desarrollo intelectual; si se continúa bajando por la diagonal de Orientación profesional se intercepta con la categoría institucional, y al bajar hacia la izquierda se encuentra que se relaciona con la variable Recursos universitarios; de esta manera se puede continuar con la lectura para la categoría socioeconómica.

4.2. El MODElo CONCEPTUAL. Considerando los resultados de las investigaciones anteriores, el modelo conceptual que se propone incorpora tanto el marco teórico como las variables que son factibles de introducir para explicar el fenómeno de la deserción en las universidades chilenas.

En la figura 2 se presenta el modelo conceptual, en el cual la deserción y permanencia es el resultado del grado de motivación que poseen los estudiantes. Si la motivación es positiva (+) el estudiante aumenta su intención por permanecer en la universidad; en cambio, si el estudiante disminuye su motivación (-), aumenta la probabilidad de desertar. El nivel de motivación cambia durante los años de estudio, pues se relaciona directamente con la integración académica y la integración social.

La integración académica se ve afectada por las características preuniversitarias y características institucionales. Las variables que constituyen las características preuniversitarias son: dependencia administrativa del colegio, rama educacional del establecimiento de origen, nota promedio de la enseñanza media, puntaje promedio de la prueba de selección universitaria (PSU), edad de egreso de la enseñanza media, edad de ingreso a la universidad y preferencia por la carrera. Las variables que se incorporan en las características institucionales son: grados académicos del cuerpo académico, condición de acreditación de la carrera, carga académica, rendimiento académico, vinculación externa, becas y créditos de financiamiento de estudios, infraestructura, satisfacción de los servicios estudiantiles y de la relación académico-estudiante.

La integración social es afectada por las características familiares y características individuales. En las características familiares se identifican las variables: número de integrantes del grupo familiar, nivel educacional de los padres, situación laboral de los padres, ingreso económico familiar, lugar de residencia (cercanía con la universidad) y valores personales (familiares y socioculturales). Las variables que constituyen las características individuales son: edad género, estado civil, situación laboral, horas de trabajo, compromiso inicial con la carrera, compromiso con metas parciales, satisfacción con la relación de pares, calidad de salud, técnicas y habilidades de estudio. Vale señalar que existe una relación importante entre las características familiares e individuales (Llinares et al. 2001).

El estudiante continuamente evalúa los resultados al superponer la integración académica y social. Esta zona en la cual se integran los ámbitos académicos y sociales es afectada por las expectativas laborales, las cuales reúnen variables asociadas al crecimiento económico del país, tasa de empleabilidad de la carrera, pertinencia laboral, nivel de remuneraciones y calidad de los ambientes laborales.

El modelo conceptual propuesto asume que todas las características actúan en forma permanente sobre el estudiante durante sus años estudios, por lo cual el estudiante está 
sometido a una tensión continua entre estos factores, manteniendo así un equilibrio en la intención de permanecer en la carrera; cuando se rompe este equilibrio, el estudiante abandona la carrera o la universidad o la educación terciaria.

\section{Figura 2}

Modelo conceptual de la deserción estudiantil

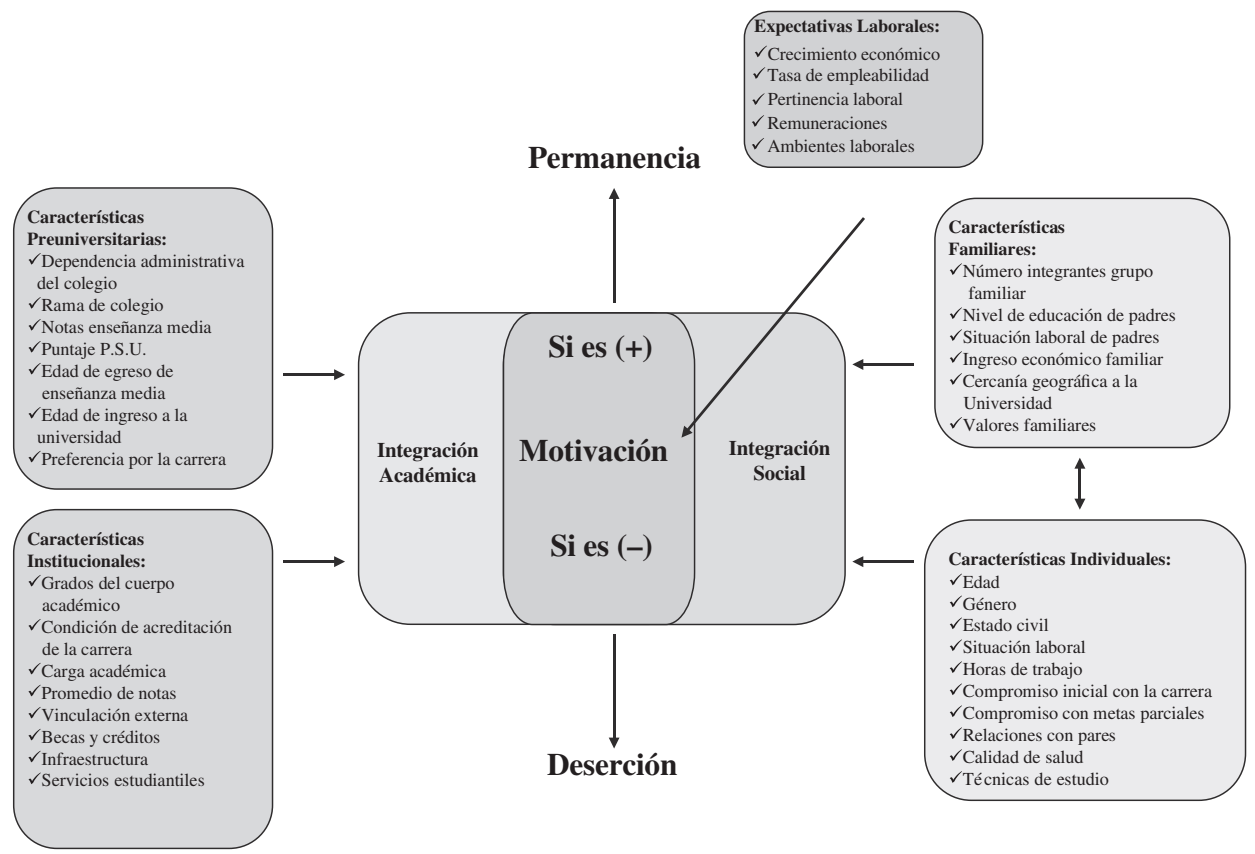

Fuente: Elaboración propia.

En la figura 3 se presenta un modelo complementario del equilibrio dinámico centrado en el estudiante de permanencia en la universidad. La construcción de este modelo se basa en el modelo conceptual anterior (fig. 2), considera las distintas categorías y variables utilizadas en los estudios revisados, los que fueron agrupados en tres ejes que mantienen este equilibrio, que son los factores: académicos, institucionales y sociales. Como el foco está centrado en el estudiante (E), en él se encuentran las características individuales, de las cuales algunas se pueden adaptar en función de la motivación que éste posea para mantenerse en la universidad. Entre ellas, están: situación laboral, compromiso inicial con la carrera y con metas parciales, técnicas de estudio, calidad de salud y relación con pares. 


\section{Figura 3}

Modelo de equilibrio dinámico centrado en el estudiante de permanencia en la universidad

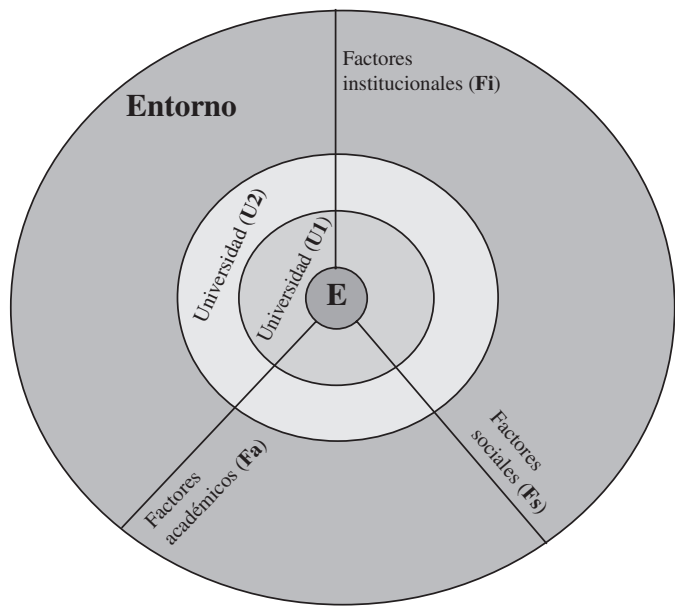

Fuente: Elaboración propia.

Los ejes se circunscriben dentro de un marco de referencia (círculo mayor) que es el entorno, entendiéndose como el medio externo al sistema de educación superior. Luego se tiene un círculo medio, correspondiente a la Universidad 2 (U2) y otro de menor radio que corresponde a la Universidad 1 (U1), en la cual actualmente se encuentra el estudiante. La diferencia entre estas dos universidades (U1 y U2) está relacionada con las características institucionales referidas a los niveles de complejidad de las mismas ${ }^{8}$. Los ejes corresponden a los factores que afectan la deserción/permanencia, y están compuestos por distintas variables relacionadas. En el caso del eje de factores académicos ( $\mathrm{Fa}$ ), las variables que lo constituyen son: rendimiento académico, carga académica, compromiso con la carrera, calidad de la carrera, actividades extracurriculares relacionadas con su formación profesional. En el eje de factores institucionales (Fi), se tiene: procesos de admisión, calidad del cuerpo académico, apoyo financiero (becas y crédito), servicios estudiantiles y calidad del programa. El tercer eje de factores sociales (Fs) comprende: actitud hacia los pares, habilidades comunicacionales, valores culturales, apoyo familiar, apoyo de pares, estrato socioeconómico y expectativas laborales.

El foco (E) puede moverse dentro de cualquier sector del círculo U1, para lo cual debe mantenerse unido a los ejes, los cuales deben cambiar su longitud frente a cambios en los otros dos ejes. Es decir, si uno de los ejes se debilita, el foco se moverá forzando a los otros ejes a reconfigurarse, para así mantener el equilibrio dinámico (mantenerse dentro de la universidad U1).

8 Para este estudio se entiende por complejidad de las universidades la modalidad en que se integran la investigación, docencia y extensión. 
Este modelo permite visualizar gráficamente la dependencia entre los factores para mantener el equilibrio dinámico. Es claro que cuando una institución acepta estudiantes con desventajas académicas se hace responsable de proveer los recursos estudiantiles que permitan, en el estudiante, mantener la intención de permanecer en la universidad U1.

Para ampliar distintas condiciones del equilibrio dinámico, en la figura 4 se presentan tres situaciones en las que el estudiante $(\mathrm{E})$, se ha trasladado de su situación teórica mostrada en la figura 3.

\section{Figura 4}

Movimientos del equilibrio dinámico (Fa: Factores académicos; Fi: Factores institucionales; Fs: Factores sociales; U1: Universidad 1; U2: Universidad 2)

a)

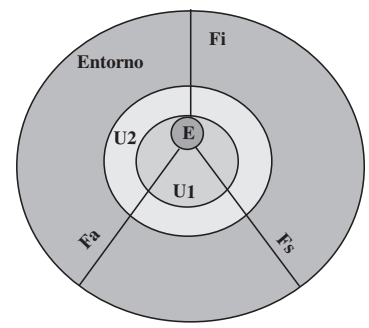

b)

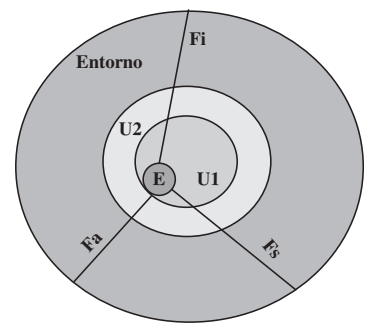

c)

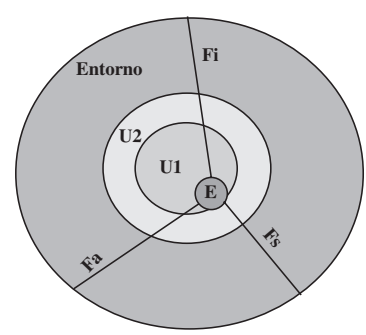

En el caso a), se ha debilitado el eje correspondiente a los factores institucionales (Fi). Ello ha generado que el estudiante reconfigure algunas variables asociadas a los factores sociales (Fs) y académicos $(\mathrm{Fa})$ para permanecer en esta nueva condición de equilibrio en la U1.

En el caso b), se encuentran debilitados los factores académicos $(\mathrm{Fa}) \mathrm{y}$ para mantenerse en la U1 el estudiante debe adaptar las variables relacionadas con los factores institucionales (Fi) y sociales (Fs).

En el caso c), se encuentran debilitados los factores sociales (Fs). Luego, para mantener el equilibrio al interior de la U1, se deben fortalecer algunas de las variables asociadas a los factores académicos $(\mathrm{Fa})$ e institucionales $(\mathrm{Fi})$.

Fuente: Elaboración propia. 
Sin embargo, cuando la universidad U1 no es capaz de responder a las necesidades de sus estudiantes, se genera un desequilibrio en el estudiante, lo que podría mover al estudiante a la universidad U2, como se observa en la figura 5a. En este caso los factores institucionales correspondientes a U1, como, por ejemplo, ayudas financieras, no son suficientes a las necesidades del estudiante. Entonces éste evalúa desertar de U1 y pasar a la U2, en la medida que encuentre respuestas a sus demandas económicas.

En el caso de la figura 5 b, cuando los factores institucionales se han debilitado fuertemente (como por ejemplo ausencia de ayudas económicas, falta de calidad de las carreras, baja relación con pares), los ejes correspondientes a los factores académicos y sociales se pueden haber reconfigurado, pero no lo suficiente para mantener el equilibrio en la universidad U2, por lo cual el estudiante rompe el equilibrio inicial y decide desertar del sistema de educación superior. En este caso, el estudiante opta por una actividad que reporte mayor beneficio en el corto plazo que permanecer en el sistema de educación terciario.

\section{Figuras $5 a$ y $b$}

Factores institucionales debilitados a) Deserción de U1 e ingreso a U2,

b) Deserción del sistema educacional terciario

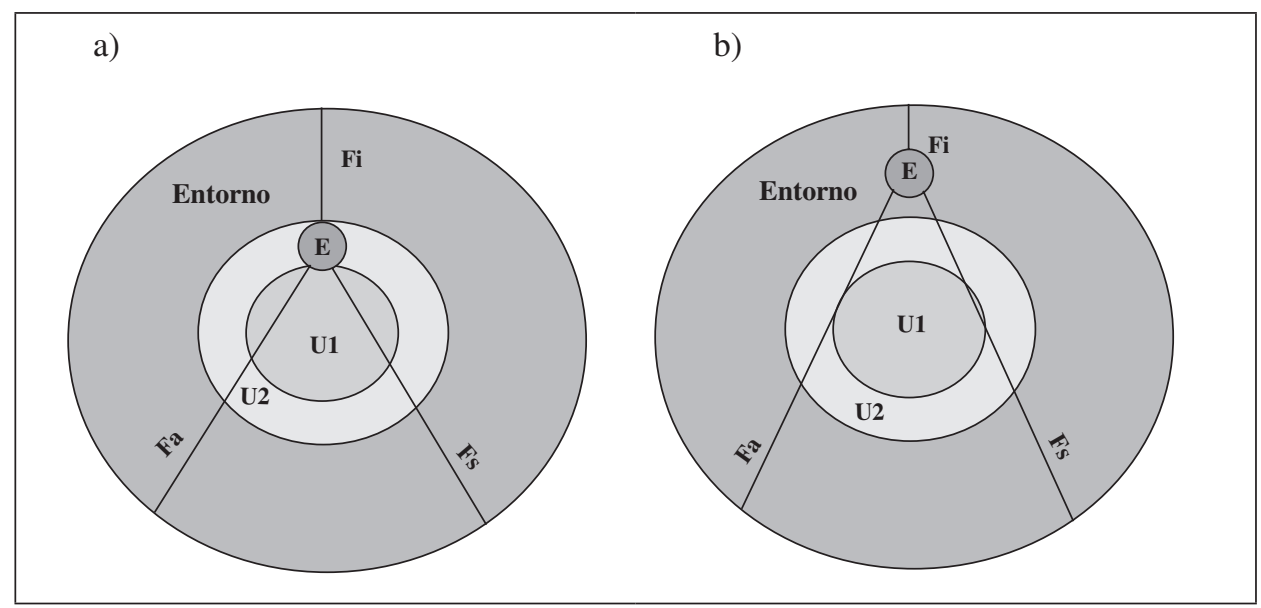

Fuente: Elaboración propia.

En los resultados se observa un equilibrio dinámico que se genera debido a la capacidad de la institución para entregar el apropiado nivel de los servicios de apoyo, para responder a las debilidades que se pueden presentar en los distintos factores que convergen en el estudiante (E).

La fortaleza del modelo de equilibrio dinámico es que permite situarse en distintos puntos de una situación base de equilibrio teórica (figura 4) a situaciones reales como los casos de las figuras 5. Este modelo ayuda a entender cómo las decisiones de desertar y 
permanecer en la universidad que realizan los estudiantes se ven afectadas por variables a través del tiempo, y cómo la universidad se ve implicada en los procesos de permanencia a través de los distintos recursos que pone al servicio de los estudiantes. En la medida que las instituciones analicen los datos históricos tanto fijos como variables de los estudiantes, podrán identificar las distintas acciones sobre estas estructuras teóricas.

El modelo tiene la capacidad de incorporar toda la historia previa del estudiante, incluidas en las características preuniversitarias, individuales y familiares; y desde un punto de vista práctico, este enfoque puede ayudar a anticipar acciones de intervención previo al ingreso de los estudiantes a la universidad; es decir, durante su permanencia en el colegio.

Para las instituciones la capacidad de aprender desde las historias estudiantiles es uno de los desafíos permanentes que deben enfrentar, ya que esto es una oportunidad de conocer y unir las aspiraciones con que llegan los estudiantes a la misión y objetivos institucionales. Con esta información, la institución puede crear y ajustar acciones de intervención focalizadas a las necesidades específicas del estudiante. El mayor desafío se presenta en la etapa de transición (primeros meses), en la cual los estudiantes calibran sus expectativas acerca de la institución y lo que realmente es la institución.

\section{CONCLUSIONES Y EXTENSIONES}

La deserción en los estudiantes es el resultado de la combinación y efecto de distintas variables. En éstas se encuentran características preuniversitarias, institucionales, familiares, individuales y las expectativas laborales. Estas influyen en la integración social y académica, las cuales a través del grado de motivación del estudiante provocan un efecto positivo -aumentando la probabilidad de permanecer- o negativo - presentando mayor probabilidad de desertar.

El modelo conceptual de deserción/permanencia propuesto permite proveer a administradores de la educación superior el marco para construir un plan de retención de estudiantes incorporando las necesidades individuales de sus estudiantes. Esto fue diseñado para que este modelo conceptual permita a administradores universitarios dedicar tiempo para realizar el seguimiento y evaluación permanente de las variables que afectan la integración social y académica, de manera de implementar estrategias de intervención focalizadas, de cara a la disminución de la deserción estudiantil.

El estudiante mantendrá una condición de equilibrio (permanencia) al combinar los factores académicos, sociales e institucionales en constante fortalecimiento de uno $\mathrm{u}$ otro factor. Si uno de los factores se debilita, para mantener el estudiante su equilibrio, es necesario que los otros factores sean capaces de reaccionar para suplir los aspectos necesarios para mantener el equilibrio inicial.

Asimismo, reporta a las instituciones de educación la necesidad de que sus factores sean tales que favorezcan la permanencia del estudiante y, con el fin de mejorarla, debe estar alerta respecto a los factores académicos y sociales del estudiante. Lo anterior, permite que en la gestión universitaria se consideren la dinámica de estos factores en el tiempo de permanencia y se comprendan las condiciones necesarias para que un estudiante no deserte de ella. 
Como extensión, el presente modelo conceptual propuesto puede ser utilizado como base para una aproximación empírica a través de modelos de duración, que permita predecir la permanencia o deserción estudiantil. Lo anterior, requiere recopilar de manera sistemática información sobre aspectos individuales, académicos, socioeconómicos de los estudiantes. Además, es posible profundizar los aspectos relacionados con las expectativas asociadas a autoeficacia y de desempeño y a los factores motivacionales relacionados con compromisos con metas académicas y de logro. Las expectativas y los factores motivacionales cambian durante su permanencia en la universidad. Por un lado, el seguimiento sistemático de éstos permitirá a los administradores universitarios orientar la gestión institucional a las necesidades de su propia población de estudiantes $\mathrm{y}$, por otro, generar acciones de intervención que provoquen cambios en los factores de riesgo y así disminuir la deserción estudiantil.

\section{BIBLIOGRAFIA}

Adelman, C. (1999). Answers in the Tool Box: Academic Intensity, Attendance Patterns, and Bachelor's Degree Attainment. Washington, DC: U.S. Department of Education. 141 pp.

Anderson, K. L. (1981). Post-high school experiences and college attrition. Journal Sociology of education. Vol. 54, $\mathrm{N}^{\circ}$ 1: 1-15.

Andrés, L., C. Hawkey, and C. Andruske (1996). Activating voices within: Individual/institutional dynamics of the first year student experience. Paper prepared for the annual meeting of the American Educational Research Association, New York.

Astin, A. (1975). Preventing student from dropping out. San Francisco: Jossey-Bass Publishers, $162 \mathrm{pp}$.

Astin A. (1999). Student Involvement: A Developmental Theory for Higher Education: Journal of College Student Development. Vol. 40, N ${ }^{\circ}$ 5: 518-529.

Bean, J. (1980). Student Attrition, Intensions and Confidence: Research in Higher Education. No 17: 291-320.

Bean, J. P. (1982). Conceptual Models of Student Attrition. In: E. T. Pascarella (Ed.). New Directions for Institutional Research: Studying Student Attrition, San Francisco USA: Jossey-Bass. $\mathrm{N}^{\circ} 36: 17-28$.

Bean, J. P. and B. S. Metzner (1985). A conceptual model non-traditional undergraduate student attrition. Review of Educational Research. Vol. 55, № 4: 485-540.

Bean, J. P. and N. Vesper (1990). Qualitative approaches to grounding theory in data: Using LISREL to develop a local model and theory of student attrition. Communication presented in the annual meeting of the American Educational Research Association, Boston, Ma.

Bean, J. P. and S. Eaton (2001). The psychology underlying successful retention practices. Journal of College Student Retention Research, Theory \& Practice Vol. 3, $\mathrm{N}^{\circ}$ 1: 73-89.

Berger, J. and J. Milem (2000). Organizational Behavior in Higher Education and Student Outcomes. In: J. Smart (Ed.), Higher Education: Handbook of theory and research. Vol. 15: 268-338.

Berger, J. (2002). Understanding the Organizational Nature of Student Persistence: Empiricallybased Recommendations for Practice. Journal of College Student Retention: Research, Theory and Practice. Vol. 3, $\mathrm{N}^{\circ}$ 1: 3-21.

Benjamin, M., and A. Hollings (1995). Toward a theory of student satisfaction: An exploratory study of the Quality of student life. Journal of College Student Development. Vol. 36, Nㅜㄴ 574- 586.

Bernal, E., A. Cabrera and P. Terenzini (2000). The relationship between race and socioeconomic status (SES): Implications for institutional research and admissions policies. Removing Vestiges: 
Research-based strategies to promote inclusion. A publication of the American Association of Community Colleges. $\mathrm{N}^{\circ}$ 3: 6-19.

Bernasconi, A. y F. Rojas (2004). Informe sobre la Educación Superior en Chile: 1980-2003. Santiago de Chile: Editorial Universitaria. 206 pp.

Braxton, J. and E. Brier (1989). Melding organizational and interactional theories of student attrition: A path analytic study. Review of Higher Education. Vol. 13, $\mathrm{N}^{\circ}$ 1: 47-61.

Braxton, J., A. Sullivan and R. Johnson Jr. (1997). Appraising Tinto's theory of college student departure. In: J.C. Smart (Ed.), Higher education: Handbook of theory and research. New York. USA. pp. 107-164.

Brunner, J. J. (2005). Educación en Chile: El Peso de las Desigualdades. En VV.AA., Conferencias Presidenciales de Humanidades. Santiago de Chile: Presidencia de la República.

Brunner, J. J. (2006). Sistema privatizado y mercados universitarios: competencia reputacional y sus efectos. Informe elaborado en el marco del proyecto FONDECYT N ${ }^{\circ} 1050138$.

Cabrera, A., A. Nora and M. Castañeda (1992). The role of finances in the persistence process: A structural model. Research in Higher Education. Vol 33, $\mathrm{N}^{\circ}$ 5: 303-336.

Cabrera, A., A. Nora and M. Castañeda (1993). College Persistence: Structural Equations Modelling Test of Integrated Model of Student Retention. Journal of Higher Education. Vol. 64, $\mathrm{N}^{\circ}$ 2: 123-320.

Cabrera, L., J. Bethencourt, P. Alvarez and M. González (2006). The dropout problem in University Study. Revista electrónica de investigación y evaluacion educativa. Vol. 12, 2.

Canales, A. y D. de los Ríos (2007). Factores explicativos de la deserción universitaria. Revista Calidad en la Educación, Ministerio de Educación de Chile. № 26: 173-201.

Carr, S. (2000). As distance education comes of age, the challenge is keeping the student. Chronicle of Higher Education. Vol. 46, $\mathrm{N}^{\circ}$ 23: A39-A41.

Casanova, D. (2005). Deserción estudiantil en la educación superior: estudio de caso en la Universidad Católica de la Santísima Concepción. Tesis presentada a la Facultad de Educación de la UCSC para optar al grado de Magíster en Ciencia de la Educación. 127 pp.

Collen, A. and W. Gasparski (1995). Design \& Systems: General Applications of Methodology (Praxiology). Transaction Publishers, U.S. 480 pp.

Dohmen, D. (2000). Vouchers in higher education. A practical approach. Paper presented at the ECER 2000-Conference, 20-23 September 2000, Edinburgh. FiBS-Forum No 4, Cologne.

Donoso, S. y E. Schiefelbein (2007). Análisis de los modelos explicativos de retención de estudiantes en la universidad: una visión desde la desigualdad social. Estudios Pedagógicos, Vol. 33, $\mathrm{N}^{\circ}$ 1: 7-17.

Durkheim, E. (1951). Suicide: A study in sociology (G. Simpson, Ed. J.A. Spaulding \$ G Simpson, Trans.). New York: Free Press.

Eccles, J. P., T. Adler and J. Meece (1984). Sex differences in achievement: A test of alternate theories. Journal of Personality and Social Psychology. Vol. 46, $\mathrm{N}^{\circ}$ 1: 26-43.

Ethington, C. (1990). A psychological model of student persistence. Research in Higher Education. $\mathrm{N}^{\circ}$ 31, Vol. 31: 279-293.

Fishbein, M. and I. Ajzen (1975). Attitudes toward objects as predictors of simple and multiple behavioural criteria. Psycological Review. $\mathrm{N}^{\circ}$ 81: 59-74.

Frankola, K. (2001). Why online learners dropout. Workforce Management. Vol. 80: 53-58.

Girón, L. y D. González (2005). Determinantes del rendimiento académico y la deserción estudiantil, en el programa de economía de la Pontificia Universidad Javeriana de Cali. Rev. Economía, Gestión y Desarrollo. Nㅜ 3: 173-201.

Gómez, B., L. Osorio, P. Domínguez, M. Silva, D. Navea, M. Monárdez y A. Silva (2002). Programa de desarrollo académico para alumnos de primer año. Revista de la Calidad de la Educación. $\mathrm{N}^{\circ}$ 17: 251-267.

González, L. y D. Uribe (2002). Estimaciones sobre la repitencia y deserción en la educación superior chilena. Consideraciones sobre sus implicancias. Revista de la Calidad de la Educación. $\mathrm{N}^{\circ}$ 17: 75-90. 
González, L. E. (2005). Estudio sobre la repitencia y deserción en la educación superior chilena. Digital Observatory for higher education in Latin America and The Caribbean. IESALCUNESCO.

Herzog, S. (2004). Measuring determinants of student return vs Dropout/Stopout vs Transfer: A first-to-second year analysis on new freshmen. In: Research in Higher Education, Vol. 46, $\mathrm{N}^{\circ}$ 8: 883-928.

Himmel, E. (2002). Modelos de análisis de la deserción estudiantil en la educación superior. Revista Calidad de la Educación. Consejo Superior de Educación. Ministerio de Educación, Chile. $\mathrm{N}^{\circ}$ 17: 91-108.

Indicadores, Números y Datos sobre Instituciones y Carreras de Educación Superior, I.N.D.I.C.E.S. (2006). Consejo Superior de Educación, Ministerio de Educación de Chile.

Ishitani, T. and S. DesJardins (2002). A longitudinal investigation od dropout from college in the United States. Journal of College Student Retention, Vol. 4, № 2: 173-201.

Ishitani, T. and T. Snider (2006). Longitudinal Effect of Collage Preparation on College Retention. Association for Institutional Research. Vol. 9, 16 pp.

Johnson, D. R. (1991). Formulating a conceptual model of nontraditional student attrition and persistence in post-secondary vocational education programs. Berkeley, CA: National Center for Research in Vocational Education. $81 \mathrm{pp}$.

Kember, D. (1989). A Longitudinal-Process Model of Drop-Out from Distance Education. The Journal of Higher Education, Vol. 60, $\mathrm{N}^{\circ}$ 3: 278-301.

Kuh, G. (2002). Organizational culture and student persistence: prospects and puzzles. Journal of College Student Retention. Vol. 3, $\mathrm{N}^{\circ}$ 1: 23-39.

Llinares, L., M. A. Molpeceres y G. Misita (2001). La autoestima y las prioridades personales de valor. Un análisis de sus interrelaciones en la adolescencia. Anales de psicología, Vol. $17, \mathrm{~N}^{\circ}$ 2: $189-200$.

Mella, J., L. Menke y J. Babul (2002). Formación universitaria en un mundo globalizado y cambiante: El programa académico de bachillerato de la Universidad de Chile. Revista de la Calidad de la Educación. № 17: 187-196.

Montes, H. (2002). La transición de la educación media a la educación superior, Retención y movilidad estudiantil en la educación superior: calidad en la educación, pp. 269-276. Publicación del Consejo Superior de Educación. Santiago.

Nye, J. (1976). Independence and Interdependence. Foreign Policy. Spring, $N^{\circ}$ 22: 130-161.

Ozga, J. and L. Sukhmandan (1998). Undergraduate non-completion: developing an explanatory model. Higher Education Quarterly. Vol. 52, No 3: 316-333.

Pascarella, E. and D. Chapman (1983a). Validation of a theoretical model of college withdrawal: Interaction effects in a multi-institutional sample. Research in Higher Education. Vol. 19, $\mathrm{N}^{\circ} 1: 25-48$.

Pascarella, E. and D. Chapman (1983b). A multi-institutional, path analytic validation of Tinto's model of college withdrawal. American Educational Research Journal. Vol. 20, $\mathrm{N}^{\circ} 1$ : $87-102$.

Pascarella, E., J. Smart and C. Ethington (1986). Long-term persistence of two-year college students. Research in Higher Education. Vol. 24, $\mathrm{N}^{\circ}$ 1, pp. 47-71.

Pascarella, E. (1985). College environmental influences on learning and development: A critical review and synthesis. In: J. C. Smart (Ed.), Higher education: Handbook of theory and research. Vol. 1. New York: Agathon.

Pascarella, E. and P. Terenzini (1980). Predicting freshman persistence and voluntary dropout decisions from a theoretical model. Journal of Higher Education. Vol. 51, $\mathrm{N}^{\circ}$ 1: 60-75.

Pascarella, E. and P. Terenzini (1991). How College Affects Students: Findings and Insights from Twenty Years of Research. San Francisco: Jossey-Bass Inc. 917 pp.

Powell, R., C. Conway and L. Ross (1990). Effect of student predisposing characteristics on student success. Journal of Distance Education. Vol. 5. № 1: 20-37. 
Prieto, A. (2002). Indecisión vocacional: pérdidas y 'perdidos' en la educación superior. Revista de la Calidad de la Educación. № 17: 145-163.

Roberts, D. (1984). Ways and means of reducing early dropout rates. Journal of Distance Education. Vol. 4, $\mathrm{N}^{\mathrm{o}}$ 1: 50-71.

Salinas, A. and J. Llanes (2003). Student Attrition, Retention, and Persistence: The case of the University of Texas Pan American. Journal of Hispanic Higher Education, Vol. 2, No 1: 73-97.

Spady, W. (1970). Dropouts from higher education: An interdisciplinary review and synthesis. Interchange. Vol. 19, $\mathrm{N}^{\circ}$ 1: 109-121.

St. John, E., A. Cabrera, A. Nora and E. Asker (2000). Economic influences on persistence. In: J. M. Braxton. Reworking the student departure puzzle: New theory and research on college student retention. Nashville: Vanderbilt University Press. pp. 29-47.

Sweet, R. (1986). Student dropout in distance education: An application of Tinto's Model. Distance Education. Vol. 7, $\mathrm{N}^{\circ}$ 2: 201-213.

Tinto, V. (1975). Dropout From Higher Education: A Theoretical Synthesis of Recent Research, Journal of Higher Education. N $\mathrm{N}^{\mathrm{T}}$ 45: 89-125.

Tinto, V. (1982). Limits of theory and practice of student attrition. Journal of Higher Education. Vol. 3, $\mathrm{N}^{\circ}$ 6: 687-700.

Tinto, V. (1987). El abandono de los estudios superiores: una nueva perspectiva de las causas del abandono y su tratamiento. Universidad Nacional Autónoma de México, Asociación Nacional de Universidades e Instituciones de Educación Superior. 55 pp.

Tinto, V. (1989). Definir la deserción: una cuestión de perspectiva. Revista de Educación Superior $\mathrm{N}^{\circ} 71$, ANUIES, México.

Tinto, V. (1993). Leaving College: Rethinking the Causes and Cures of Student Attrition, $2^{\mathrm{a}}$ Edition, Chicago: University of Chicago Press. 246 pp.

UNESCO (2004). Repetition at high cost in Latin America and the Caribbean. IESALCUNESCO.

Voorhees, R. (1987). Toward building models of community college persistence: A logit analysis. Research in Higher Education. Vol. 26, $\mathrm{N}^{\circ} 2$ : 115-129.

Wang, J., E. Wu and P. Wong (2004). Strategies for Reducing Dropout from Distance Education Courses. In G. Richards (Ed.), Proceedings of World Conference on E-Learning in Corporate, Government, Healthcare, and Higher Education 2004 (pp. 1705-1710).

Wylie, J. (2005). Non-Traditional Student Attrition in Higher Education: A theoretical model of separation, disengagement then dropout. SELF Research Centre, University of Western Sydney, Australia. WYL05439. 12 pp. 